\title{
Five cases of paroxysmal kinesigenic dyskinesia by genetic diagnosis
}

\author{
GUO-HONG CHEN \\ Department of Neurology, Zhengzhou Children's Hospital, Zhengzhou, Henan 450053, P.R. China
}

Received April 9, 2014; Accepted October 28, 2014

DOI: $10.3892 /$ etm.2014.2155

\begin{abstract}
Paroxysmal kinesigenic dyskinesia (PKD) is an autosomal dominant disorder and PRRT2 is the causative gene of PKD. The aim of this study was to investigate PRRT2 mutations in patients who were clinically diagnosed with PKD. Nine PKD cases, including four familial cases and five sporadic cases, were selected. Peripheral blood was drawn after obtaining informed consent, and genomic DNA was extracted by a standard protocol. Sanger sequencing was performed for the screening of PRRT2 mutations. A total of five cases were detected to harbor PRRT2 mutations. Four familial cases carried a c.649dupC (p.Arg217Profs*8) mutation, while one sporadic case and his asymptomatic father carried a c.133-136delCCAG (p.Pro45Argfs*44) mutation. PRRT2 mutations were not identified in the remaining cases. The study further confirmed that PRRT2 was a causative gene of PKD and implied that PRRT2 mutation has incomplete penetrance.
\end{abstract}

\section{Introduction}

Paroxysmal kinesigenic dyskinesia (PKD), also known as paroxysmal exercise-induced dyskinesia syndrome, is an autosomal dominant genetic disease, the incidence rate of which is $\sim 1 / 150,000$ (1). The clinical features are sudden exercise-induced dance-like movements of the limbs or torso, throwing disorders, stiffness or numbness, dystonia and other actions (2). PKD is mostly onset in childhood and increases in prepubescence; however, the symptoms are spontaneously relieved in adulthood and disappear at an age of 30-40 years (3). The patients have clear consciousness at onset, and exhibit normal electroencephalograms during seizures and the interictal period, which differentiates it from epilepsy. Patients with PKD are very sensitive to antiepileptic drugs; small doses of carbamazepine or phenytoin can

Correspondence to: Dr Guo-Hong Chen, Department of Neurology, Zhengzhou Children's Hospital, 255 Gangdu Street, Zhengzhou, Henan 450053, P.R. China

E-mail: guohongchencn@126.com

Key words: paroxysmal kinesigenic dyskinesia, PRRT2, incomplete penetrance effectively control the seizures of PKD, and the symptoms may disappear completely in certain patients after treatment with drugs for three days (4).

Since it was first reported in 1967 (5), it has been confirmed that there are two pathogenic gene regions in PKD, specifically 16p11.2-q12.1 and 16q13-q22.1 (6,7). There may be a third pathogenic region in PKD; however, this is not on chromosome 16 (8). Nevertheless, the causative gene has not been cloned. In 2011, Chen et al collected eight families with PKD and discovered using whole exon sequencing combined with Sanger sequencing that all eight families were carrying PRRT2 gene mutations, while no PRRT2 mutations were found in the 1,000 cases of the normal control group (9). Therefore, Chen et al identified PRRT2 as a causative gene of PKD, which was confirmed in subsequent studies (10-12). However, there is little literature concerning PKD, and genetically confirmed cases of PKD are rarely reported. In the present study, nine cases of clinically diagnosed PKD were collected from 2007 onwards, and the genes of these patients were sequenced by the Sanger method. The incidence of PRRT2 mutations in the patients was determined to investigate PRRT2 as a pathogenic gene in PKD.

\section{Materials and methods}

Subjects. The study included nine patients with PKD (cases PKD 1-9) from seven different pedigrees (families 1-7). These included six male cases (PKD 2, 3, 5 and 7-9) and three female cases (PKD 1, 4 and 6). Four patients had a family history (PKD 1-4) and the other five were sporadic cases. Family 1 had eight members, three of whom had a history of PKD, but the patient 1 refused a blood sample and thus it was not possible to determine whether that patient carried PRRT2 mutations. The family 2 proband exhibited involuntary twisting of the body and his father mainly complained of sudden movement. The remaining five probands and their parents were asymptomatic and classified as sporadic cases. The present study was approved by the Ethics Committee of Zhengzhou Children's Hospital (Zhengzhou, China). All patients provided informed consent.

Blood sample collection. Venous blood samples $(3 \mathrm{ml})$ were obtained for anticoagulation by sodium citrate. They were stored in a refrigerator at $4^{\circ} \mathrm{C}$ for short periods, and frozen at $-20^{\circ} \mathrm{C}$ for long-term preservation. 
Table I. PRRT2 gene forward and reverse primers.

\begin{tabular}{lll}
\hline Exons & \multicolumn{1}{c}{ Forward primers $\left(5^{\prime} \rightarrow 3^{\prime}\right)$} & \multicolumn{1}{c}{ Reverse primers $\left(5^{\prime} \rightarrow 3^{\prime}\right)$} \\
\hline Exons 1 & TTGCCTGGGTAACGCGTGGCT & ACACCCGCATTCCCGTGCAGT \\
Exons 2a & CAATT GGGCCTGCAGTGCTGAG & GGTTTGGACACTGTTTCTTGGCAT \\
Exons 2b & GGAGGGGAATCAAAGGCCAACTG & TCAACCAGCTGCTGCAGCACTC \\
Exons 2c & GAAAAGCAAGAGAATGGGGCAGTG & GATTACTCCAGAGGCTCTATTGCAG \\
Exons 3-4 & TTCTGGATGACTTTTCCACCTGAT & CAACAGGAAGAAAAGTCTTGGGAT \\
\hline
\end{tabular}

DNA extraction. The QIAamp blood DNA extraction kit (Qiagen, Hilden, Germany) was used to extract DNA from the blood samples. The DNA was dissolved with $1 \mathrm{X}$ AE solution (Qiagen), and stored at $-20^{\circ} \mathrm{C}$ for the long-term.

Polymerase chain reaction (PCR). Five PRRT2 primers were used according to the literature (9), and are shown in (Table I). Four exons including exon-intron joints ( $\geq 20 \mathrm{bp}$ ) were amplified. The PCR annealing temperature was $66^{\circ} \mathrm{C}$ (exon 1) or $60^{\circ} \mathrm{C}$ (exon $\left.2 \mathrm{a}, 2 \mathrm{~b}, 2 \mathrm{c}, 3-4\right)$.

Sequencing. To $5 \mu \mathrm{l}$ PCR product was added $0.3 \mu 1$ shrimp alkaline/heat-sensitive alkaline phosphatase [Promega (Beijing) Biotech Co., Ltd., Beijing, China], $0.2 \mu \mathrm{l}$ exonuclease [New England Biotechnology (Beijing) Co., Ltd., Beijing, China] and $2.5 \mu 1 \mathrm{ddH}_{2} \mathrm{O}$ for purification. The mixture was placed in a $37^{\circ} \mathrm{C}$ water bath for $1 \mathrm{~h}$, then in a $80^{\circ} \mathrm{C}$ water bath for $15 \mathrm{~min}$. The $3 \mu \mathrm{l}$ purified PCR product that was obtained was added to $1 \mu 13.2 \mathrm{pmol} / \mathrm{l}$ unidirectional primer and $1 \mu \mathrm{l}$ BigDye working fluid (Beijing Yue Wei Gene Technology Co., Ltd., Beijing, China) for forward sequencing reaction. The reaction conditions were: $96^{\circ} \mathrm{C}$ denaturation for $1 \mathrm{~min} ; 94^{\circ} \mathrm{C}$ denaturation for $10 \mathrm{sec}, 50^{\circ} \mathrm{C}$ annealing for $5 \mathrm{sec}$ and $60^{\circ} \mathrm{C}$ extension for $4 \mathrm{~min}$, for $25 \mathrm{cycles}$; followed by $60^{\circ} \mathrm{C}$ extension for $10 \mathrm{~min}$. Following the sequencing reaction, $50 \mu 175 \%$ ethanol mixture was added and the resultant mixture was stored at $-20^{\circ} \mathrm{C}$ in a refrigerator for $60 \mathrm{~min}$. After centrifuging at $10,800 \mathrm{x} \mathrm{g}$ for $30 \mathrm{~min}$, the supernatant was discarded, $50 \mu 175 \%$ ethanol was added and further centrifugation at $10,800 \mathrm{x}$ g for $30 \mathrm{~min}$ was conducted. The supernatant was discarded. After drying, $7 \mu \mathrm{l}$ Hi-Di (Shanghai Top Zhuo Biotechnology Co., Ltd., Shanghai, China) was added to the residue, which was the loaded onto an ABI 3730 genetic Analyzer (Haimai Pu Biotechnology Co., Ltd.) for sequencing.

\section{Results}

Clinical manifestations. The clinical manifestations of the nine patients with PKD are shown in Table II, and the familial pedigrees of five of the cases are shown in Fig. 1. The proband (PKD 2) in family 1 had the chief complaint of involuntary limb swinging when starting to run that was automatically relieved after lasting for $\sim 30 \mathrm{sec}$. The consciousness of the patient was clear at onset and the seizures occurred 3-10 times per day. The proband's mother (PKD 1) reported dance-like movements of the upper limbs at the age of 10 years; however, the symptoms were mild and improved without treatment. Questioning revealed that the proband's grandfather had

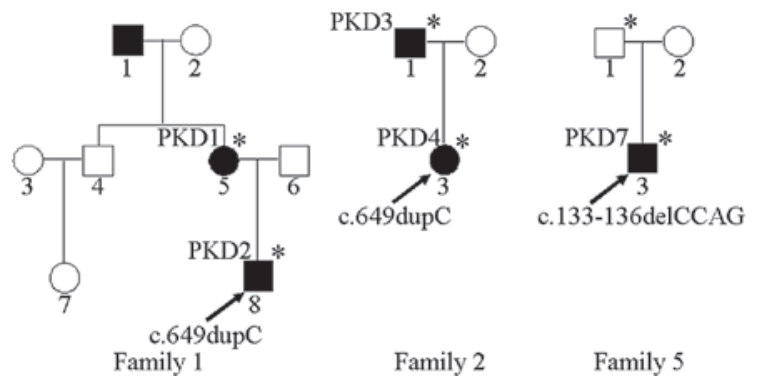

Figure 1. Pedigree chart for the patients with paroxysmal kinesigenic dyskinesia (PKD). $\square$ indicates male, $\circ$ represents female, $\square$ or $\bullet$ denotes affected

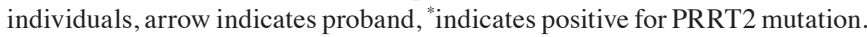

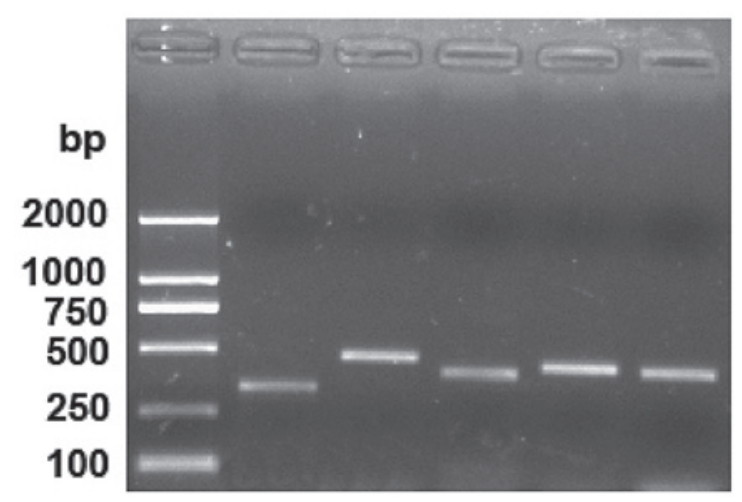

Figure 2. PCR gel results of four PRRT2 gene exons. M, DNA marker; lanes 1-5: exon 1 , exon $2 \mathrm{a}$, exon $2 \mathrm{~b}$, exon $2 \mathrm{c}$ and exons $3-4$, respectively.

a history of dance-like movements of the upper limbs. The proband (PKD 4) in family 2 involuntarily stood from a seated position with a twisted body posture and strange facial expressions, and the father (PKD 3) had similar attacks at the ages of 10-18 years but no longer experienced symptoms. Sporadic case PKD 5 had double-leg spasms in sudden movements, each episode lasting for $\sim 10$ sec. PKD 6 and PKD 9 had lower limb stiffness in a sudden movement or tensing, which was sustained for 5-15 sec; PKD 9 sometimes fell down. PKD 7 experienced involuntary limb swinging in sudden movements. PKD 8 had left upper limb spasticity in sudden movements or when changing position.

PRRT2 gene sequencing. A gel map following PCR amplification is shown in Fig. 2. The amplification bands of the five PCR primers were $329,467,398,433$ and 431 bp respectively.

Sanger sequencing results showed that PKD 1-4 carried the PRRT2 gene c.649dupC (p.Arg217Profs*8) mutation (Fig. 3A) 


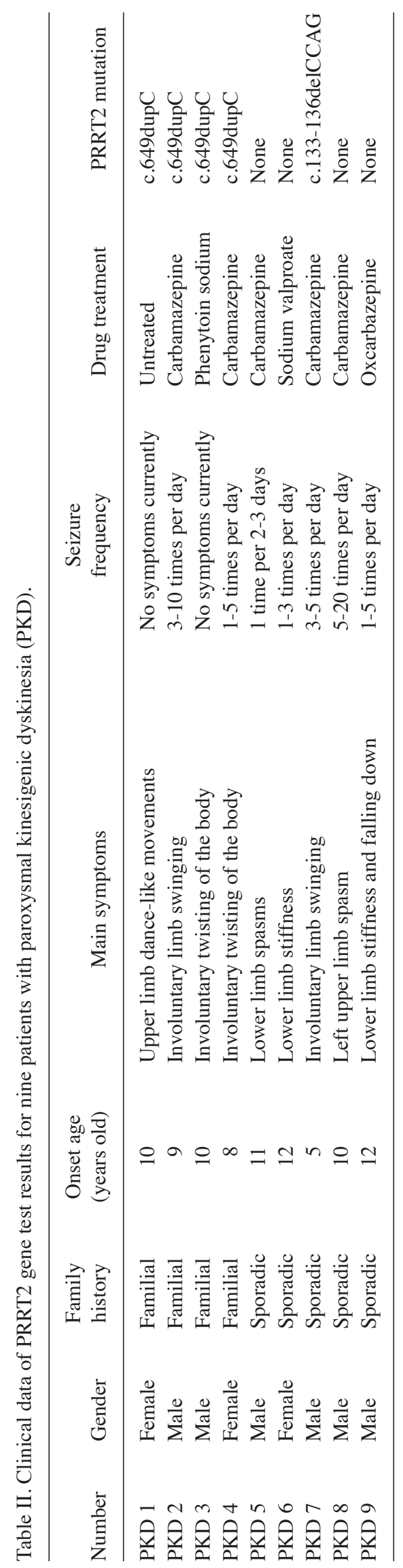

A

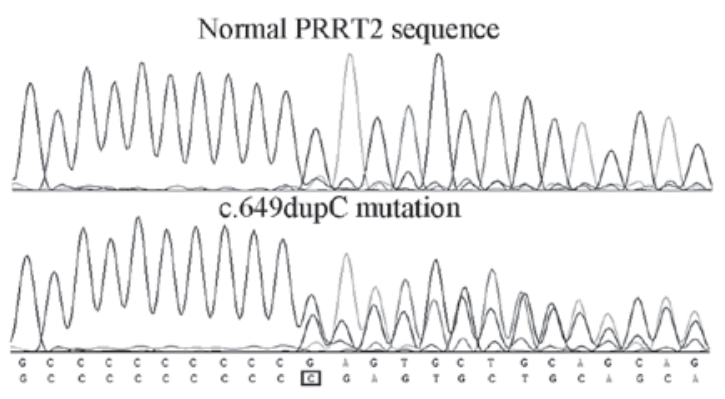

B

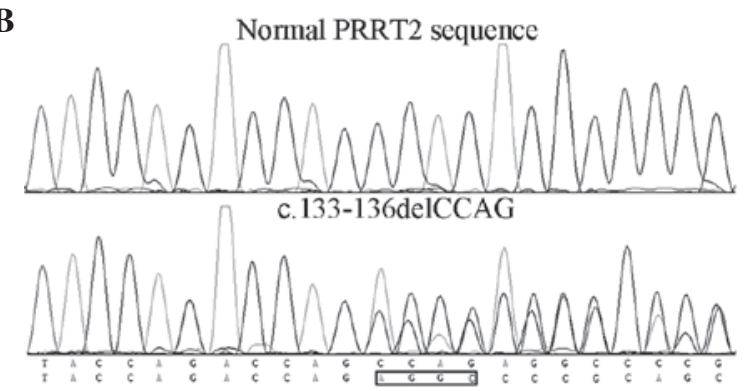

Figure 3. Normal PRRT2 and (A) c.649dupC and (B) c.133-136delCCAG mutated gene sequences.

and PKD 7 carried the c.133-136delCCAG (p.Pro45Argfs*44) mutation (Fig. 3B), while no PRRT2 gene mutations were identified in the remaining patients. Since PKD 7 was a sporadic case, further screening for the mutation was performed and it was found that the asymptomatic father also carried the mutation.

\section{Discussion}

PKD is the most common form of paroxysmal dyskinesia with onset triggered by sudden movements. Since PKD was first reported in 1967, a number of academics and clinicians have been committed to identifying the genes that cause PKD. In 2011, Chen et al reported internationally that PRRT2 was a PKD disease-associated gene (9), and this was soon confirmed by other research groups (10-12). It has now been confirmed that PRRT2 is a causative gene of PKD. The findings of Chen et al provided the foundation for the clinical molecular diagnosis of PKD.

However, few studies have reported on PKD. Song and $\mathrm{Hu}$ described in detail the clinical manifestations of five PKD cases (13); Li et al reported seven PKD patients (14) and Lin et al reported three PKD pedigrees with clinical and genetic characteristics (15). Mao et al reported the clinical features of 34 PKD patients (16). However, PKD cases confirmed by genetic methods were not reported. In the present study, nine cases with a clinical diagnosis of PKD were collected, including four cases with a family history. It was found that all four cases with familial PKD and one case with sporadic PKD carried PRRT2 mutations by Sanger sequencing of the PRRT2 gene, which further confirmed that PRRT2 is a causative gene of PKD. In family 5, the proband PKD 7 carried a PRRT2 gene mutation at the c.133-136delCCAG site; however, further testing found that the asymptomatic father of PKD 7 also carried the same genetic mutation. This suggests that PRRT2 mutations have incomplete penetrance, that is, that certain 
individuals carrying the gene mutations of PKD do not exhibit clinical symptoms. The reason for the incomplete penetrance of PRRT2 gene mutations may be influenced by the environment or other genetic modifications.

Currently, $>30$ PRRT2 mutations have been identified in patients with PKD (9-12,17-21), of which $>95 \%$ are truncated mutants, while only $\sim 5 \%$ are missense mutations and splicing mutations (22). In the PRRT2 gene mutations, c.649dupC is the most common site, accounting for $\sim 80 \%$ of cases (19). The high incidence of the mutations has prompted c.649dupC to be defined as a high-frequency mutation. In the present study, four of the five patients carrying PRRT2 mutations had c.649dupC mutations, accounting for $80 \%$, which confirms the high frequency of this mutation. The study by Li et al found that c.649dupC mutations can be de novo mutations (23), suggesting that the mutation was not from a founder effect but was a hotspot mutation. Other studies support that c.649dupC is a hotspot mutation $(18,19)$.

Although PRRT2 genes of PKD have been cloned, the function of PRRT2 remains unclear. Co-immunoprecipitation results suggest that PRRT2 interacts with SNAP25 $(12,24)$. SNAP25 is a presynaptic membrane protein involved in the docking and fusion of synaptic vesicles and associated with the release of neurotransmitters (25). Since SNAP25 forms a SNARE complex with syntaxin and synaptobrevin (26), this suggests that PRRT2 may have functional connectivity with the SNARE complex, or may be a component of the SNARE complex. In addition, SNAP25 induces synaptic vesicles to release neurotransmitter when triggered by $\mathrm{Ca}^{2+}$, indicating that PRRT2 mutations are likely to cause abnormalities in neurotransmitter release (27). Whether PRRT2 mutations affect intracellular $\mathrm{Ca}^{2+}$ influx remains unclear, and requires further investigation.

The present study confirmed that PRRT2 is a pathogenic gene of PKD, and that c.649dupC is a high frequency mutation site. In addition, the presence of incomplete penetrance of PRRT2 was revealed.

\section{References}

1. Bennett LB, Roach ES and Bowcock AM: A locus for paroxysmal kinesigenic dyskinesia maps to human chromosome 16 . Neurology 54: 125-130, 2000.

2. Demirkiran M and Jankovic J: Paroxysmal dyskinesias: clinical features and classification. Ann Neurol 38: 571-579, 1995.

3. Bhatia KP: Paroxysmal dyskinesias. Mov Disord 26: 1157-1165, 2011.

4. Bruno MK, Hallett M, Gwinn-Hardy K, et al: Clinical evaluation of idiopathic paroxysmal kinesigenic dyskinesia: new diagnostic criteria. Neurology 63: 2280-2287, 2004.

5. Kertesz A: Paroxysmal kinesigenic choreoathetosis. An entity within the paroxysmal choreoathetosis syndrome. Description of 10 cases, including 1 autopsied. Neurology 17: 680-690, 1967.

6. Tomita H, Nagamitsu S, Wakui K, et al: Paroxysmal kinesigenic choreoathetosis locus maps to chromosome 16p11.2-q12.1. Am J Hum Genet 65: 1688-1697, 1999.
7. Valente EM, Spacey SD, Wali GM, et al: A second paroxysmal kinesigenic choreoathetosis locus (EKD2) mapping on 16q13-q22.1 indicates a family of genes which give rise to paroxysmal disorders on human chromosome 16. Brain 123: 2040-2045, 2000.

8. Spacey SD, Valente EM, Wali GM, et al: Genetic and clinical heterogeneity in paroxysmal kinesigenic dyskinesia: evidence for a third EKD gene. Mov Disord 17: 717-725, 2002.

9. Chen WJ, Lin Y, Xiong ZQ, et al: Exome sequencing identifies truncating mutations in PRRT2 that cause paroxysmal kinesigenic dyskinesia. Nat Genet 43: 1252-1255, 2011.

10. Li J, Zhu X, Wang X, et al: Targeted genomic sequencing identifies PRRT2 mutations as a cause of paroxysmal kinesigenic choreoathetosis. J Med Genet 49: 76-78, 2012.

11. Wang JL, Cao L, Li XH, et al: Identification of PRRT2 as the causative gene of paroxysmal kinesigenic dyskinesias. Brain 134: 3493-3501, 2011

12. Lee HY, Huang Y, Bruneau N, et al: Mutations in the gene PRRT2 cause paroxysmal kinesigenic dyskinesia with infantile convulsions. Cell Rep 1: 2-12, 2012.

13. Song $\mathrm{F}$ and $\mathrm{Hu} \mathrm{ZY}$ : Clinical features and pathogenesis of paroxysmal dyskinesias. J Clin Neurol (China) 18: 382-384, 2005 (In Chinese).

14. Li SY,Liu WP, Xiong K and Xiao B: Paroxysmal exercise-induced dyskinesia (clinical report of 7 cases). Stroke Nerv Dis 13: 48-49, 2006 (In Chinese).

15. Lin $\mathrm{Y}, \mathrm{Wu} \mathrm{ZY}$, Wang $\mathrm{N}$ and Murong SX: The clinical and genetic features of familial paroxysmal kinesigenic dyskinesia:the three families reports. Zhonghua Shen Jing Ge Za Zhi 39: 734-737, 2006 (In Chinese).

16. Mao CY, Shi CH, Song B, et al: Genotype-phenotype correlation in a cohort of paroxysmal kinesigenic dyskinesia cases. J Neurol Sci 340: 91-93, 2014

17. Méneret A, Grabli D, Depienne C, et al: PRRT2 mutations: a major cause of paroxysmal kinesigenic dyskinesia in the European population. Neurology 79: 170-174, 2012.

18. Cao L, Huang XJ, Zheng L, Xiao Q, Wang XJ and Chen SD: Identification of a novel PRRT2 mutation in patients with paroxysmal kinesigenic dyskinesias and c.649dupC as a mutation hot-spot. Parkinsonism Relat Disord 18: 704-706, 2012.

19. Lee YC, Lee MJ, Yu HY, et al: PRRT2 mutations in paroxysmal kinesigenic dyskinesia with infantile convulsions in a Taiwanese cohort. PLoS One 7: e38543, 2012.

20. van Vliet R, Breedveld G, de Rijk-van, Andel J, et al: PRRT2 phenotypes and penetrance of paroxysmal kinesigenic dyskinesia and infantile convulsions. Neurology 79: 777-784, 2012.

21. Li HF and Wu ZY: PRRT2 mutations and PRRT2 disorders. Human Genet Embryol 3: 105, 2013.

22. Heron SE and Dibbens LM: Role of PRRT2 in common paroxysmal neurological disorders: a gene with remarkable pleiotropy. J Med Genet 50: 133-139, 2013.

23. Li HF, Ni W, Xiong ZQ, Xu J and Wu ZY: PRRT2 c.649dupC mutation derived from de novo in paroxysmal kinesigenic dyskinesia. CNS Neurosci Ther 19: 61-65, 2013.

24. Stelzl U, Worm U, Lalowski M, et al: A human protein-protein interaction network: a resource for annotating the proteome. Cell 122: 957-968, 2005.

25. Jarvis SE and Zamponi GW: Masters or slaves? Vesicle release machinery and the regulation of presynaptic calcium channels. Cell Calcium 37: 483-488, 2005.

26. Sørensen JB, Matti U, Wei SH, et al: The SNARE protein SNAP-25 is linked to fast calcium triggering of exocytosis. Proc Natl Acad Sci USA 99: 1627-1632, 2002.

27. Prescott GR, Gorleku OA, Greaves J and Chamberlain LH: Palmitoylation of the synaptic vesicle fusion machinery. J Neurochem 110: 1135-1149, 2009. 